\title{
Magnetic measurements with a SQUID magnetometer: Possible artifacts induced by sample-holder off-centering
}

\author{
Nieves Casañ-Pastor, ${ }^{\text {a),b) }}$ Pedro Gomez-Romero, ${ }^{\text {b) }}$ and Louis C.W. Baker ${ }^{\text {a }}$ \\ Chemistry Department, Georgetown University, Washington, DC 20057
}

\begin{abstract}
Asymmetries in sample holders used for magnetic measurements on magnetometers based on induction methods, such as those equipped with SQUID sensors, can lead to substantial errors and/or important artifacts which resemble phase transitions. They occur under the conditions for which sample and/holder have signals of opposite sign, but are nearly equal in magnitude. The most serious errors can occur often, but not exclusively, for compounds having intermediate magnetic dilution. We present here a general discussion of the problem illustrated by measurements of a polyoxometallate with the known Keggin structure $\mathrm{K}_{6}\left[\mathrm{Fe}\right.$ (III) $\left.\mathrm{W}_{12} \mathrm{O}_{40}\right] \cdot n \mathrm{H}_{2} \mathrm{O}$, done on a SHE VTS model 905 . While one of the obvious solutions to this problem is the use of holders with a signal much smaller than that of the sample, it would be preferable if, in addition, the holder had a response of the same sign as that of the sample, for all temperatures and fields measured.
\end{abstract}

\section{INTRODUCTION}

A word of caution is given here about routine magnetic measurements with magnetometers based on the induction method, particularly SQUID magnetometers, which, in view of their high sensitivity, are now widely used.

Although the symmetry requirements for sample holders are usually mentioned in the instructions for the instrument, the extent to which small deviations from perfect symmetry can affect the output data for each particular sample is not generally stated. In most cases, such errors will be less than $\sim 10 \%$ of the susceptibility data. However, in all those cases where the magnetic response of the sample changes considerably with respect to the holder signal, as a function of temperature or field, and is compensated at some point by the latter, an artifact resembling a phase transition can result. Typical cases would be compounds of intermediate magnetic dilution, for example, heteropoly anions or metal clusters. The highly temperature- and field-dependent signals of ferromagnets or superconductors could also be subject to this artifact.

\section{DESCRIPTION OF THE INSTRUMENT AND DATA ACQUISITION}

Several good descriptions of SQUID (superconducting quantum interference device) magnetometers are found in the literature. ${ }^{1-6}$ The instrument used by us was a VTS model 905 from SHE. The system consists of a superconducting pickup loop in a uniform magnetic field. If the magnetic sample is introduced into the loop, there is a resulting magnetic- flux change proportional to the susceptibility of the sample. This is detected by a sensor consisting of a superconducting ring with a single Josephson function coupled to a if circuit (SQUID). The pickup loop may consist of two coils instead of one, counterwound, so that instrument drifts and external magnetic noise will af-

\footnotetext{
a) Authors to whom correspondence should be addressed.

b) Present address: Institut de Ciencia de Materials de Barcelona, CSIC, Martí i Franqués s/n, 08028 Barcelona, Spain.
}

fect them in opposite ways. The change in current flowing in the superconducting ring appears as an output voltage proportional to the current. Once the instrument is calibrated, the output voltage is converted to absolute magnetization. ${ }^{1}$ A diagram of the coil geometry of this particular susceptometer is given in Fig. 1(a). It consists of a pair of pickup coils at a nominal distance of $5.28 \mathrm{~cm}$ between each other's centers. ${ }^{7}$ There are two kinds of operating modes, "continuous" and "peak to peak." In the continuous mode, the sample is kept steadily centered in one of the coils, and relative changes of sample magnetization can be followed. This operating mode does not cancel any error coming from the instrument shifts or ambient magnetic noise, and its use is not recommended above a temperature of about $120 \mathrm{~K}^{8}$
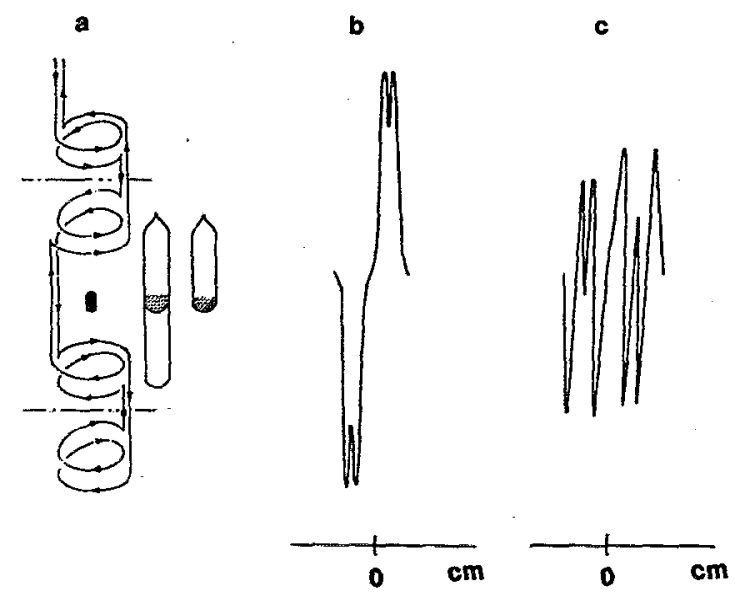

FIG. 1. Coil geometry and experimentâl voltage outputs typical in a SQUID magnetometer. (a) Coil geometry in case of a double pickup loop. The sample is oscillated from the midpoint between coils $(l=0$ $\mathrm{cm}$ ), up passing the upper one, back through the center, passing the lower one, and back to center. (b) Optimal voltage output ( $V$ vs $1 \mathrm{~cm}$ from center), found also in asymmetric cases when the signal of the sample is much bigger in magnitude than the signal of the holder. (c) Voltage output for off-centered holder and sample, at the temperature at which both signals compensate. 
In the peak-to-peak mode, the sample is moved cyclically from one coil to the other with a span slightly larger than the actual separation of the coils (Fig. 1). Maximum output is reached when the sample passes through one of the coils. Because the coils are counterwound, a maximum signal of opposite polarity is obtained when the sample passes through the second coil, and it is this maximum difference in signals what the sensor measures. ${ }^{2}$

If the sample is long enough so that neither of the coils can feel the end of it (in the peak-to-peak mode), the output voltage should be proportional to the volume susceptibility of the sample. ${ }^{5}$ On the other hand, if the sample is a point dipole (a sample $0.6 \mathrm{~cm}$ long can be considered so), the response of the instrument is proportional to the magnetization of the sample.

Since the amount of sample is usually limited, this last type of measurement is the most widely used. Thus, the ideal way to measure is to attach the sample to the end of a piece of nonmagnetic thread and oscillate it between both coils. This is not possible for powder or liquid samples, which need a holder. The signal observed, then, is a combination of sample and holder signals. Keeping the sample length and diameter less than $0.6 \mathrm{~cm}$, to fulfill the pointdipole approximation, there is no restriction on the length of the holder as long as it is possible to correct for its contribution to the total signal (and the cycling does not take it to the bottom of the instrument).

\section{DISCUSSION}

Figure 1(b) represents a typical experimental voltage output when a sample is oscillated between the coils with a span bigger than the separation between coils. The sensor takes the maximum flux excursion while the sample is cycling between both coils and divides by $2,{ }^{2}$ thus, that sample has to be centered correctly between the coils (avoiding a residual flux in excess in one of them), which is achieved easily at a temperature at which its signal is high, and so any variation off-center is detected. If the center of gravity of the sample and holder do not match, the contribution from the latter to the total signal will not be equal to the one obtained when the holder was measured empty (that is, when the holder was centered itself). This could be corrected, in principle, by knowing how much the holder is off-center and so what percent of the holder signal is contributing on top of the maximum signal from the sample.

Figures 1(b) and 1(c) show experimental voltages upon sample cycling for a system where the sample and sample-holder signals have opposite signs (para- and diamagnetic, respectively) and their centers of gravity are separated (off-centered) at least $3 \mathrm{~mm}$. When the signal from the sample is much bigger than that of the holder, such output closely resembles the correct one [Fig. 1(b)], and it is not possible to detect any asymmetry. However, once both signals become close in magnitude (for example, upon changing the temperature or field), a confusing output results [Fig. 1(c)].

In order to understand what is really shown in the measurement control unit, we have simulated global voltage outputs by combining signals from the sample and

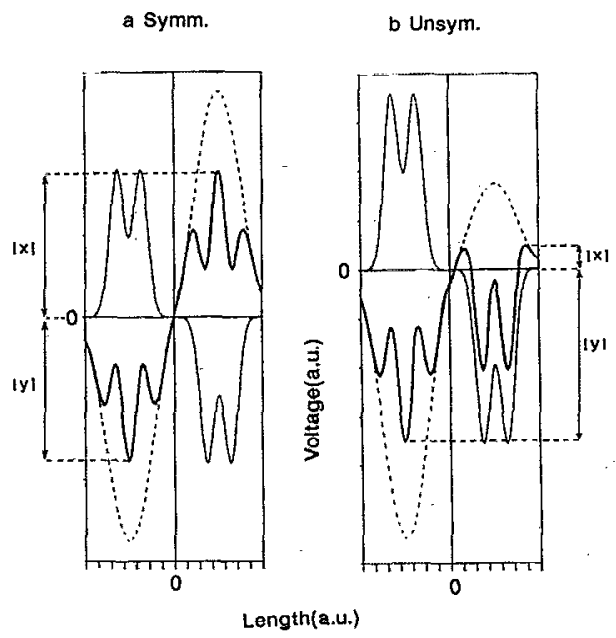

FIG. 2. Simulated voltage outputs for cases in which sample and holder give a response of the same magnitude, but opposite sign. (a) Sample and holder are perfectly centered and (b) sample and holder are off-centered about $12 \%$ of the span distance (that is, about $1 \mathrm{~cm}$ ).

holder for a case of perfect centering and a case of $1-\mathrm{cm}$ off-centering of the holder with respect to the sample (see Fig. 2). This simulation is done for a hypothetical set of conditions at which the magnitudes of both signals are equal, but they are of opposite sign. The main assumptions are that the coil response as the sample moves through it is Gaussian, and that the sample behaves as a point dipole, while the holder does not, thus yielding a broader Gaussian for the latter. Each half of the wave corresponds to the response (alternating in sign) from each of the coils, as the sample moves from the midpoint between coils (length = $0 \mathrm{~cm}$ ) toward each coil. A double maximum in response is normally obtained because the sample is oscillated between two positions past each of the coils. This is represented as two close Gaussians.

The dashed line represents the holder signal (as it would be observed if the holder was oscillated independently), and the continuous thin line represents the sample signal. The continuous thick line is the overall signal that would be observed experimentally and has been obtained by adding at each position the sample and sample-holder signals. The magnitudes of the output obtained would be $(|x|+|y|) / 2$, which are obviously different in each case. In spite of the simplicity of the simulation, it fits the observed effects well. The error caused by this asymmetry gets reduced as the sample signal becomes bigger with respect to the holder signal, or vice versa, but it does still exist.

The effects of this sort of measurement artifact can be seen in Fig. 3, which shows the results obtained for $\alpha-\mathrm{K}_{6}\left[\mathrm{Fe}^{\mathrm{III}} \mathrm{W}_{12} \mathrm{O}_{40}\right](\mathrm{I})$, a heteropoly blue ${ }^{9-11}$ with a molecular weight of 3329 , in different degrees of magnetic dilution and asymmetry of the system sample-sample holder. The values are corrected for the corresponding holder signal measured previously.

The same solid sample of (I), once run under the correct conditions (using a AlSi alloy bucket designed to 


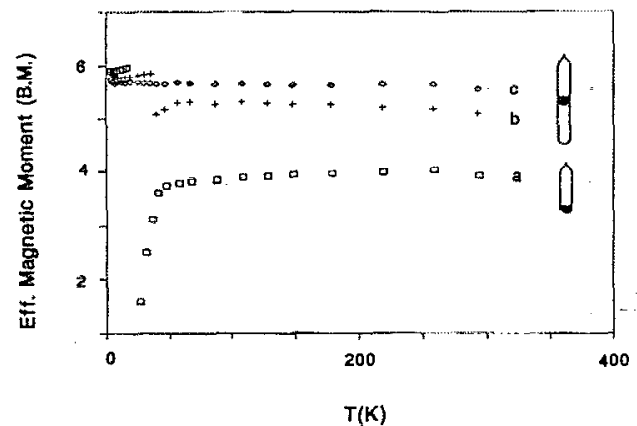

FIG. 3. Effective magnetic moment for $\mathrm{K}_{6}\left[\mathrm{Fe}\right.$ (III) $\left.\mathrm{W}_{12} \mathrm{O}_{40}\right] \cdot n \mathrm{H}_{2} \mathrm{O}$ measured in (a) $0.05-\mathrm{mol}$ solution in an asymmetric suprasil quartz holder (1.8- $\mathrm{cm}$ off-centering); (b) same solution in a quasisymmetric quartz holder; (c) $0.15 \mathrm{-g}$ solid, in same quasisymmetric holder.

avoid asymmetry), showed a Curie plot in the whole temperature range, corresponding to $5.70 \mu_{B}$. The big effect of the artifact is striking when comparing Figs. $3(\mathrm{a})$ and 3(b). But even in Fig. 3(c) the error induced is considerable above the temperature of change $\left(0.1 \mu_{B}\right.$ in this case and up to $1 \mu_{B}$ in more diluted cases). It is common to find even apparent diamagnetic signals for a paramagnetic sample when asymmetries such as that shown in Fig. 3(a) occur. On the other hand, other samples not containing $\mathrm{Fe}^{\mathrm{III}}$ but $\mathrm{V}^{\mathrm{IV}}, \mathrm{V}^{\mathrm{III}}, \mathrm{W}^{\mathrm{V}}$, or $\mathrm{Co}^{\mathrm{II}}$ tetrahedral, will still show a sharp change even in solid form. ${ }^{9}$ The holders discussed so far were made especially for our inert atmosphere requirements. The usc of standard quartz holders also yielded an artifact for solid samples of compounds similar to (I), but which are more diluted magnetically. The correlation between the "transition" temperatures for each of these compounds and their degree of magnetic dilution was the first hint of the presence of an artifact independent of the real behavior of the samples. ${ }^{9}$ Only one example has been presented in this paper, for sake of brevity.

Finally, it is important to remark that such an artifact shows up not only as a sharp transition, but also introduces an error in the remaining points, affecting them to an extent that depends on the relative value of the signals and on the degree of asymmetry. Thus, even if a false transition never shows, the magnetic data may be drastically affected.

One of the solutions to the problem exposed in this work is, of course, the use of malleable materials for the design of perfectly symmetric holders. Metal and plastic holders would be a good choice, if properly constructed.

In principle, another solution would be measuring the holder's response using the same centering between coils that it had when measured containing the sample. For cases where sample and holder have a response of the same sign, this would be the perfect solution. Howeyer, Fig. 2 shows that when sample and holder have signals of opposite sign, the measurement artifact shows only in the global signal. In such a case, correcting the experimental output with the holder run off-center would not be appropriate. It is then highly advisable to use, when possible, holders with magnetization of the same sign as that of the sample.

\section{CONCLUSIONS}

The artifact described in this paper is favored by the degree of magnetic dilution of the samples, as in the example presented. Air-sensitive samples, for which sealed holders are often needed, also represent a high-risk case, given the usual asymmetry of the sealing. It is very likely that data from many other samples, with highly variable magnetic responses, such as ferromagnets or superconductors, will be subject to a considerable error or even show a misleading jump in magnetization. It is even possible to observe a "Meissner effect," falsely suggesting superconductivity, when measuring a paramagnetic sample in a diamagnetic holder or, conversely, a false ferromagnetic type of behavior when measuring a sample that approaches diamagnetism with a paramagnetic holder.

It is most important to avoid conditions wherein the signals from holder and sample become equal or close in magnitude, but opposite in sign.

\section{ACKNOWLEDGMENTS}

We thank Dr. Ekk Sinn for making available to us the VTS magnetometer and Dr. T N Thaniasiri for help in its use. We thank Dr. X. Obradors for helpful comments. This work was supported by a Grant and two Fellowships (N.C.P. and P.G.R.) of the Spanish-American Joint Committee for Cultural and Educative Cooperation.

${ }^{1}$ E. J. Cukauskas, D. A. Vincent, and B. S. Deaver, Jr., Rev. Sci. Instrum. 45, 1 (1974).

${ }^{2}$ J. A. Good, in SQUID Superconducting Quantum Interference Devices and Their Applications, Proceedings of the Conference on Superconducting Devices, Berlin, 1976, edited by H. D. Hahlbohm and H. Lubbig (de Gruyter, New York, 1977).

${ }^{3}$ M. Cerdonio, C. Cosmelli, G.L. Romani, C. Messana, and C. Gramaccioni, Rev. Sci. Instrum. 47, 1 (1976).

${ }^{4}$ M. Cerdonio, F. Mogno, G.L. Romani, C. Messana, and C. Gramaccioni, Rev. Sci. Instrum. 48, 300, (1977).

5 J. S. Philo and W. M. Fairbank, Rev. Sci. Instrum. 48, 1529 (1977).

${ }^{\circ}$ C. J. O'Connor, B. S. Deaver, Jr., and E. Sinn, J. Chem. Phys. 70, 5161 (1979).

${ }^{7}$ SHE Cryogenics, Instrument Manual VTS 950., San Diego, CA.

${ }^{8}$ e.g. J. W. Dawson, H. B. Gray, E. Hoenig, G. R. Rossman, J. M. Schredder, and R.-H. Wang, Biochemistry. 11, 461 (1972).

${ }^{9}$ N. Casan-Pastor, Doctoral dissertation, Georgetown University, Washington, DC, May 1988, Diss. Abstr. Int. B 50, 1397-B (1989).

${ }^{10}$ Heteropoly anions retain their molecular identity and structure in solid and solution (Ref. 11).

${ }^{11} \mathrm{~L}$. C. W. Baker, in Advances in the Chemistry of Coordination Compounds, edited by S. Kirschner (Macmillan, New York, 1962), p. 604 